\title{
ABUNDANCIA, USO DE HÁBITAT Y COMPORTAMIENTO DE LA TINGUA MOTEADA (Gallinula melanops bogotensis Chapman 1914) EN EL HUMEDAL GUAYMARAL, BOGOTÁ - COLOMBIA
}

\author{
ABUNDANCE, HABITAT USE AND BEHAVIOUR OF \\ THE SPOT-FLANKED GALLINULE (Gallinula melanops \\ bogotensis Chapman 1914) IN THE GUAYMARAL \\ WETLAND, BOGOTÁ - COLOMBIA
}

\author{
Karin Osbahr ${ }^{1}$, Norma Constanza Gómez²
}

\begin{abstract}
${ }^{1}$ Líder Grupo de Investigación en Fauna Silvestre. Universidad de Ciencias Aplicadas y Ambientales U.D.C.A. Dirección para correspondencia: Calle 222 No. 55-37 Bogotá - D.C kosbahr@udca.edu.co ${ }^{2}$ Unidad de Investigación en Fauna Silvestre. Universidad de Ciencias Aplicadas y Ambientales U.D.C.A. Dirección para correspondencia: Calle 222 No. 55-37 Bogotá - D.C norvet1908@yahoo.com
\end{abstract}

Rev. U.D.C.A Act. \& Div. Cient. 14(1): 81 - 91, 2011

\section{RESUMEN}

Los humedales urbanos de Bogotá aún conservan fauna endémica entre la cual se destaca la tingua moteada (Gallinula melanops bogotensis) por ser una subespecie propia del sistema de humedales de la cordillera Oriental en los departamentos de Cundinamarca y Boyacá. Los pocos humedales que todavía subsisten en el área de Bogotá y sus alrededores han sido considerados como los ecosistemas más amenazados de Colombia y como consecuencia las poblaciones de G. $m$. bogotensis han disminuido significativamente. El objetivo del presente trabajo fue el de establecer la abundancia de la especie a través de monitoreos continuos durante tres años consecutivos, indicando a su vez los requerimientos de hábitat para mantener la especie. Se realizaron conteos directos tres veces por mes en las horas de la mañana desde enero de 2008 y marzo de 2010 para un total de 69 días de muestreo. Para complementar el estudio, se hicieron observaciones puntuales durante cuatro días completos tomando datos de la actividad. Aún cuando el humedal ha sufrido un deterioro permanente subsiste una población con actividad reproductiva. La abundancia relativa de la población mostró variaciones a lo largo de los meses, sectores y categorías de edad muestreados. Las observaciones del comportamiento de G. m. bogotensis permitieron identificar a su vez un mayor uso, reflejado en una mayor abundancia relativa, hacia los sectores que mantienen vegetación flotante, mientras que los juncales, la ronda y las asociaciones vegetales registraron menor abundancia en todos los sectores estudiados.

Palabras clave: Rallidae, preferencia, vegetación pleustofítica, poblaciones.

\section{SUMMARY}

The urban wetlands of Bogota have preserved endemic fauna among which stands out the spot-flanked Gallinule (Gallinula melanops bogotensis) as an endemis subspecies of the wetland system of the Eastern Cordillera in the departments of Cundinamarca and Boyacá. The few wetlands that still exist in the area of Bogota and its surroundings have been considered the most threatened ecosystems in Colombia and as a result the populations of G. m. bogotensis have dropped significantly. The aim of this study was to establish the abundance of the species through continuous monitoring for three consecutive years, indicating in turn the habitat requirements for maintaining the species. Direct counts were performed three times per month in the morning from January 2008 to March 2010 for a total of 69 days of sampling. To complement the study, specific observations were made during four full days taking activity data. Even if 
the wetland has suffered permanent changes a population with reproductive activity remains. The relative abundance of the population showed changes over the months, sectors and age groups sampled. The observations of the behavior of $G$. m. bogotensis showed a higher habitat use, reflected in greater abundance, relative to sectors that keep floating vegetation, while at the reeds, the round and recorded plant associations the species was less abundant in all the sectors studied.

Key words: Rallidae, preference, pleustophytic vegetation, populations.

\section{INTRODUCCIÓN}

Los humedales de la sabana de Bogotá se caracterizan por la presencia de cuerpos de agua o zonas de inundación y las zonas de transición o ronda hidráulica, originando un ecosistema heterogéneo del cual se beneficia particularmente la avifauna. Estos ecosistemas ofrecen refugio, alimento, zonas de nidificación y ambientes importantes donde se concentran las aves residentes durante el período de muda de plumaje o las aves migratorias durante la migración anual (ABO, 2000; Becerra et al. 2004). Los humedales de planicie son considerados prioritarios para la conservación por ser parte del complejo que conforma el Área de Importancia para la Conservación de las Aves de la Sabana de Bogotá (AICA) (Scott E Carbonell, 1985; Franco E Bravo, 2005; DAMA, 2006). Sin embargo, la desecación y el relleno, la contaminación por desechos sólidos y la disminución de la calidad del agua por procesos de eutroficación, derivados de elevadas concentraciones de fósforo (Guarnizo $\mathcal{E}$ Calvachi, 2004), han deteriorado severamente este ecosistema. Los efectos antrópicos adicionalmente han modificado la fisonomía vegetal de los humedales repercutiendo en la pérdida de la cobertura vegetal original.

La elevada destrucción de los humedales ha disminuido considerablemente las poblaciones de la avifauna, afectando especialmente a la Tingua Moteada (Gallinula melanops bogotensis), subespecie que se distribuye en la cordillera Oriental de Colombia entre los 2500 y 3050m de elevación en los departamentos de Cundinamarca y Boyacá (Stiles, 1998). Junto con el descenso de las poblaciones de G. m. bogotensis se ha documentado la fragmentación de las mismas, por lo cual la especie califica en peligro crítico de extinción en Colombia (Arzuza et al. 2008; Cifuentes $\mathcal{E}$ Castillo-Cortés, 2009). Se estima que su población total es menor a los 2500 individuos (Renjifo, 1998; Cadena, 2002), y es evidente que existe una pérdida de la variabilidad genética debida a una reducción demográfica extrema (Becerra $\mathcal{E}$
Cely-Fajardo, 2007). En la Sabana de Bogotá la principal amenaza que enfrenta la especie es la desaparición de vegetación acuática y la desecación para el establecimiento de campos de cultivos y potreros (Hernández $\mathcal{E}$ Andrade, 2006). Un ejemplo claro de las potenciales consecuencias de los manejos inadecuados para la avifauna es el del humedal la Florida, en el cual, G. $m$. bogotensis fue erradicada a comienzos de la década pasada, tras el uso de herbicidas en un infortunado ensayo para eliminar el buchón de agua (Eichornia crassipes) (Cadena, 2002).

A pesar de de su condición de vulnerabilidad, el conocimiento de G. $m$. bogotensis es aún muy escaso. Aún cuando es reportada regularmente en los conteos anuales de aves, se desconocen aspectos tan importantes como la ecología de sus poblaciones, los requerimientos de hábitat y no existe un monitoreo cercano y detallado del comportamiento de esta especie (Becerra et al. 2005; Van der Hammen et al. 2008). Siendo una especie que depende totalmente de ambientes acuáticos y que no puede sobrevivir en otros ambientes y que el humedal de Guaymaral requiere de medidas de conservación urgentes, el conocimiento de los parámetros biológicos mencionados es fundamental para facilitar el desarrollo de un plan de manejo para la conservación de la especie. Por lo tanto, el objetivo del presente trabajo fue el de establecer la abundancia de la especie a través de monitoreos continuos durante tres años consecutivos, evaluando a su vez el comportamiento en relación al hábitat disponible en el humedal Guaymaral.

\section{MATERIALES Y MÉTODOS}

El humedal Guaymaral, con una extensión de 43ha, se encuentra ubicado al nororiente del casco urbano de Bogotá (Figura 1a), a 2550m de altitud presentando un clima frío distribuido en un piso bioclimático altitudinal subandino o andino bajo (Matallana et al. 2004). La pérdida de los principales afluentes tales como el canal Torca y la Quebrada El Guaco, han modificado el régimen hídrico del humedal, por lo cual el espejo de agua se presenta de manera más o menos permanente, dependiendo su extensión de los periodos de lluvia (Osbahr E Gómez, 2006). En el extremo occidental del humedal se forman estanques artificiales que en época invernal mantienen volúmenes importantes de agua (CIC, 2003). El humedal se encuentra rodeado de pastizales ubicados en zonas de relleno y destinados a la producción pecuaria, especialmente de ganado bovino y equino. El área de estudio presenta un mosaico de vegetación introducida no plantada y de crecimiento espontaneo, que ocupa tanto las zonas de ronda como los sectores inundables; áreas reforestadas con especies nativas e introducidas y áreas 


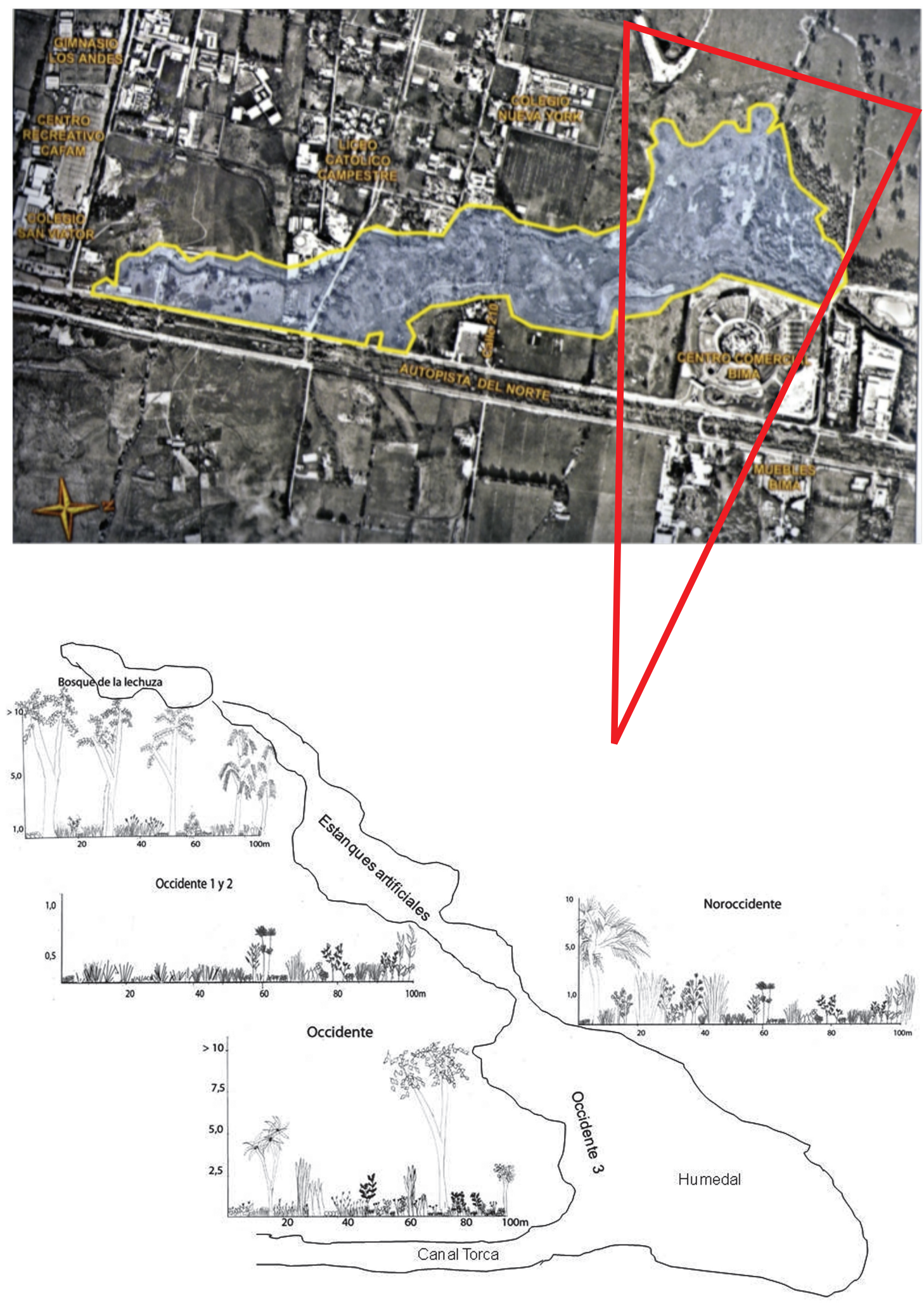

Figura 1. a. Ubicación geográfica del humedal Guaymaral, Bogotá. Fuente: Conservación Internacional - Colombia (2003); b. Esquema del área de estudio empleada entre 2008 y 2010 dentro del humedal, y descripción de la cobertura vegetal encontrada en cada uno de los sectores: sectores "Bosque de lechuza", "Noroccidente", "Occidente", "Occidente 1", "Occidente 2" y "Occidente 3". 
de vegetación nativa tanto terrestre como acuática. La vegetación flotante dominante es Eichornia crassipes, planta introducida que llega a cubrir grandes áreas del humedal (Osbahr \& Hernández-Schmidt, 2006).

Entre enero y mediados de julio de 2008 (36 días de muestreo) se dividió el área de estudio en tres sectores definidos en trabajos previos (Osbahr \& Gómez, 2006) como "Bosque de la Lechuza" y sectores "Occidente" y "Noroccidente" (Figura 1b). Considerando que los procesos antrópicos que afectan el humedal modificaron el paisaje impidiendo el muestreo en el sector "Noroccidente", se dividió, entre finales de julio de 2008 y marzo de 2010 (33 días de muestreo), el sector "Occidente" en sectores 1, 2 y 3, diferenciando así la zona del humedal de los estanques artificiales (Figura 1b). El sector "Bosque de la Lechuza" se mantuvo. Para cada sector se obtuvo un perfil de la vegetación marcando con un decámetro transectos longitudinales de 100 metros y midiendo con una cinta métrica la altura de las plantas registradas (Mostacedo E Fredericksen, 2000).

Debido a que G. m. bogotensis nada en aguas abiertas, pero también camina sobre la vegetación flotante y se refugia en los juncales (ABO, 2000), se aplicó en cada sector el método de conteo por puntos (Bibby et al. 1998). En los puntos seleccionados al azar y con un intervalo de $250 \mathrm{~m}$ entre cada punto de observación (Ralph et al. 1996), se registraron durante 10 minutos todos los individuos de la especie. Las observaciones se realizaron con binoculares $(21 \times 40)$ visitando el área de estudio semanalmente entre las 5:30 a 8:00 am, con una diferencia de seis días entre cada visita, para un total de 1656 horas de muestreo. El día escogido para los muestreos fue aleatorio. Cabe mencionar, que en algunas oportunidades el avistamiento de G. m. bogotensis disminuyó por la lluvia y la neblina o por la presencia de personas, perros o ganado bovino y equino cerca o dentro del espejo de agua. La abundancia relativa se calculó por sector cómo el número de individuos registrados respecto al esfuerzo de muestreo calculado como: horas totales $\mathrm{x}$ sectores totales/días totales. La prueba de normalidad arrojó la presencia de valores extremos por lo cual se aplicó una prueba de Kruskall-Wallis (Zar, 1999) para establecer diferencias entre los sectores muestreados. Para establecer las categorías de edad se tuvo en cuenta la descripción dada para la especie en ABO (2000) y Hilty E Brown (1986).

Para obtener datos de la actividad asociada a las diferentes comunidades vegetales, definidas a partir de los perfiles construidos para cada sector en el área de estudio (Figura 1b), se aplicaron en julio de 2009 observaciones Ad libitum (Lehner, 1996). Estas observaciones se realizaron una vez por semana completando cuatro días de muestreo con intervalos de 15 minutos entre las 5:30am y las 5:00pm. Con los comportamientos registrados se estableció el etograma de la especie (Hutt $\mathcal{E}$ Hutt, 1970). Con las observaciones comportamentales se calculó la frecuencia/hora definida como el número de veces que el ave exhibe determinado comportamiento. Las frecuencias obtenidas para cada categoría comportamental se relacionaron con el uso de la vegetación. Todos los análisis se realizaron utilizando Statgraphics Plus 5.1C.

\section{RESULTADOS Y DISCUSIÓN}

Durante todo el estudio se obtuvo un total acumulado de 1603 registros de individuos de G. m. bogotensis en diferentes estados de desarrollo (polluelos 41, jóvenes 171 y adultos 1391 registros respectivamente). Estas cifras indican que, aún cuando el humedal ha sufrido un deterioro permanente, subsiste una población con actividad reproductiva. La abundancia relativa de la población mostró variaciones a lo largo de los meses muestreados (Figura 2). Durante el año 2008 se registró una variación bimodal, con picos de máxima abundancia en julio y diciembre; en 2009 se mantuvo la tendencia fluctuante y el máximo de abundancia también correspondió al mes de julio, aunque en los demás meses se observó un descenso notable que se mantuvo hasta los primeros meses del año 2010. En general, la abundancia relativa promedio ( $\mathrm{AR}=7,73 \pm 6,1$ individuos/ esfuerzo de muestreo) de G. $m$. bogotensis en el humedal Guaymaral es mayor a la reportada para el humedal Jaboque en la localidad de Engativá (Bogotá, D.C) donde, aunque es residente, es poco común ( $A R=1$ ) (Rueda-C. et al. 2005).

La abundancia relativa total registró fluctuaciones con respecto a los sectores muestreados, siendo mayor $(\mathrm{AR}=$ 40,7 y 37,0 individuos/esfuerzo de muestreo respectivamente) en los sectores "Occidente 1 y 2 " con cuerpos de agua bien definidos (Figura 3). Observaciones anteriores corroboran los resultados obtenidos, ya que mencionan que G. m. bogotensis es la más acuática de las especies de Rálidos de la Sabana, encontrándose casi siempre nadando, aunque también camina en la vegetación flotante y se refugia en los juncales (Hilty E Brown, 1986; ABO, 2000; Restall et al. 2007; Osbahr E Wüst, 2009).

Estas fluctuaciones se observaron igualmente con respecto a la distribución de la abundancia relativa por categorías de edad por sector (Figura 3). Debido a su tamaño pequeño, la abundancia de polluelos puede estar subestimada por la presencia de juncales especialmente en los sectores "Noroccidente" y "Occidente 3". Igualmente, el avistamiento de polluelos se encuentra ligado a la actividad reproductiva de la especie, puesto que la anidación, con un período de incubación de 15 a 20 días (Lozano, 1992), al igual que los 


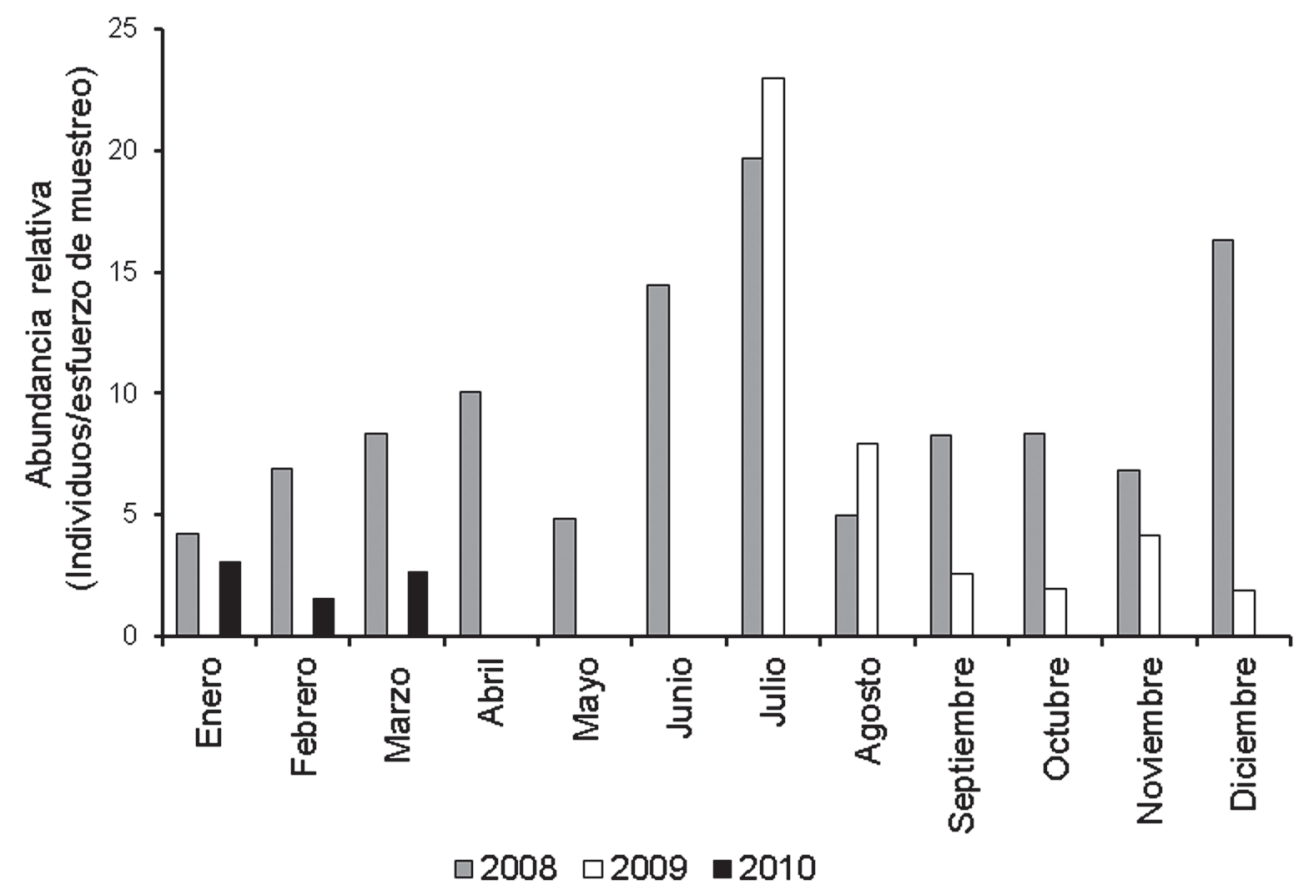

Figura 2. Fluctuación en la abundancia relativa de la población de G. m. bogotensis en el humedal Guaymaral a lo largo de los meses muestreados en cada año.

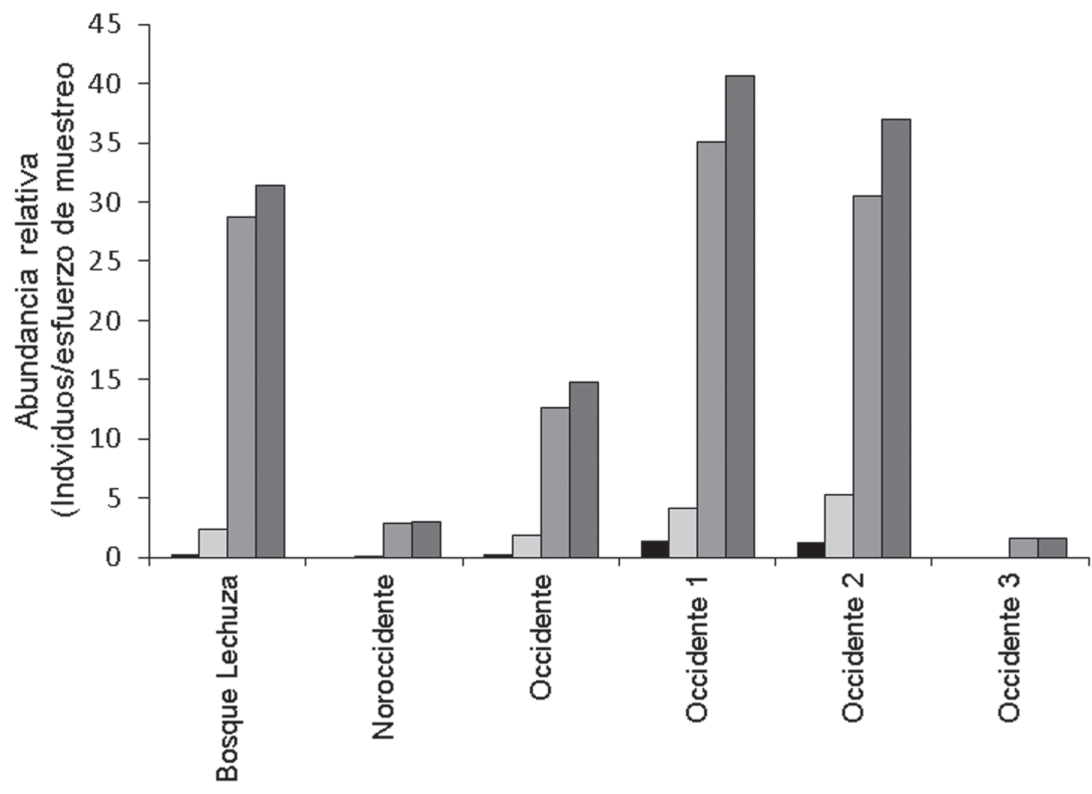

- Polluelos $\square$ Jovenes $\square$ Adultos $\square$ Total

Figura 3. Variación en la abundancia relativa por categoría de edad de G. m. bogotensis en cada sector muestreado en el humedal Guaymaral durante el estudio. Esfuerzo de muestreo total de 12,48 horas*sector/día. 
primeros días de cuidado de los polluelos, se desarrollan en zonas de elevada cobertura vegetal (Becerra et al. 2004a). En el humedal Guaymaral se observó el mayor número de polluelos (9 a 13 individuos) entre julio y octubre, mientras que en los meses de febrero, junio, noviembre y diciembre disminuyó el número (1 a 3 polluelos). Estos registros coinciden parcialmente con Becerra et al (2004a) quienes reportaron anidaciones en la laguna de Fúquene (Cundinamarca), durante marzo y mayo y un segundo periodo entre octubre y diciembre. Borrero (1956), Varty et al. (1986), Cadena (2002) y Becerra et al. (2005) afirman que la reproducción se concentra entre los meses de marzo y junio con posturas ocasionales durante el mes de septiembre. Sin embargo, de acuerdo con Zuluaga-Bonilla (2007), la especie anidaría durante todo el año en presencia de ambientes artificiales, con algunas posturas extras casi de inmediato para reponer nidadas no exitosas, derivadas de las amenazas antrópicas cercanas. En el humedal Guaymaral los individuos jóvenes fueron observados en mayor cantidad durante el segundo semestre donde se contaron entre 9 y 28 individuos; contrariamente, en los demás meses los valores obtenidos siempre fueron menores a los 10 individuos. En los meses de enero y abril no se encontraron individuos jóvenes en las diferentes zonas del humedal.

Se encontraron diferencias estadísticamente significativas al comparar las abundancias relativas de G. m. bogotensis en los diferentes sectores muestreados (Figuras $4 \mathrm{a} \mathrm{y}$ b). Esta diferencia es atribuible a la presencia de comunidades vegetales que caracterizan a cada sector, por lo cual la variabilidad en la abundancia podría reflejar el uso del hábitat de manera dinámica de acuerdo a la disponibilidad de recursos (Kusch et al. 2008). Los sectores "Noroccidente", "Occidente" y "Occidente 3" se caracterizan por conservar juncales (Typha angustifolia, Schoenoplectus californicus, Cyperus rufus, Juncus effusus y Juncus sp.) asociados con

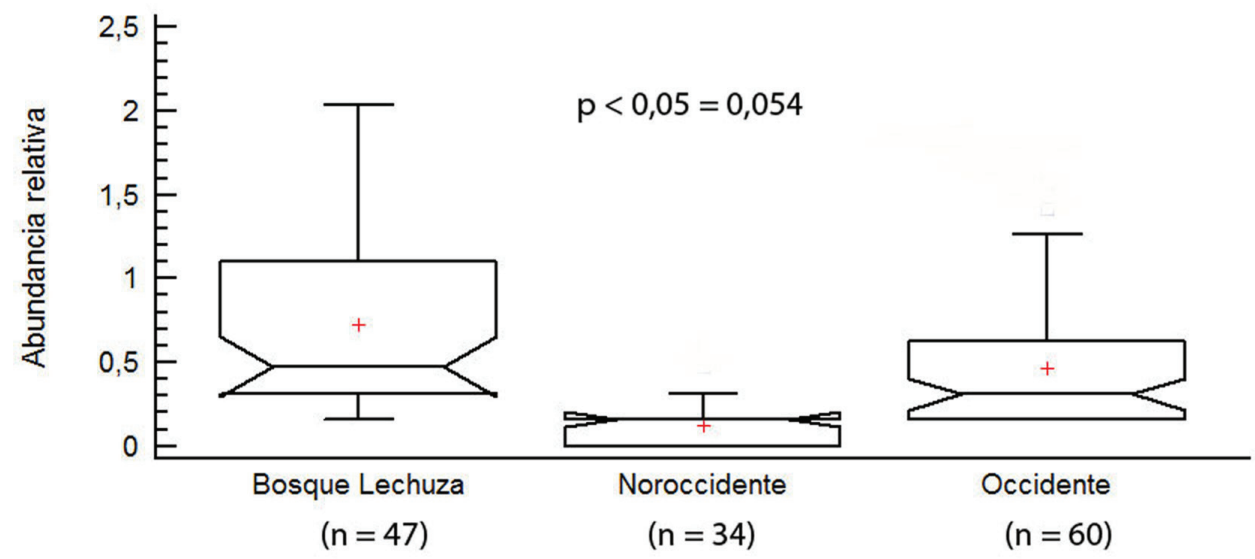

a.

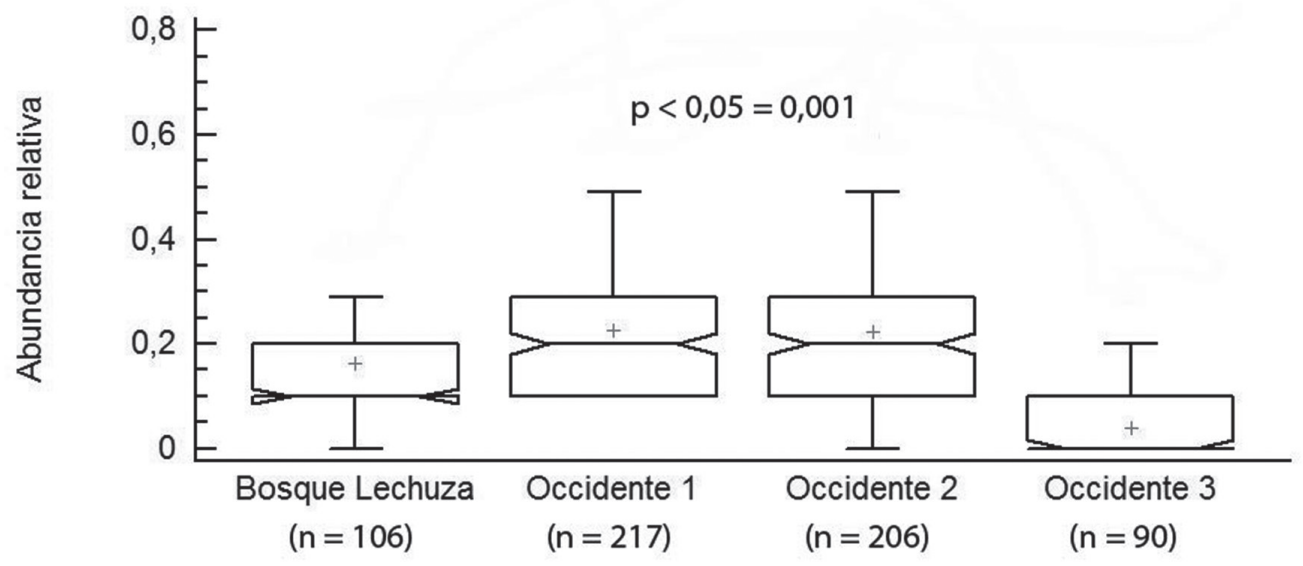

b.

Figura 4. Variación en la abundancia relativa de G. m. bogotensis en el humedal Guaymaral (Prueba de Kruskall-Wallis nivel de confianza del 95\%). 4a. Sectores muestreados entre enero y mediados de julio de 2008. 4b. Sectores muestreados entre finales de julio de 2008 y marzo de 2010. 
Polygonum sp. y Rumex sp. y una ronda con vegetación herbácea (Cirsium sp., Oxalis corniculata, Kikuyuochloa clandestina, Hyrocotile bonplandii, Verbena litoralis, Holcus lanatus, Rumex sp., Trifolium repens, Senecio madagascarensis y Polygonum sp.) (Figura 1b). En los sectores "Occidente 1 y 2", el cinturón de juncales está compuesto únicamente por $J$. effusus y $C$. rufus. En el bosque de la lechuza la cobertura de juncales es baja y el cuerpo de agua está rodeado por plantaciones mixtas de Cipreses (Cupressus sp.), Pinos (Pinus sp.), Eucaliptos (Eucalyptus sp.) y Sauces (Salix humboldtiana) (Figura 1b). Sin embargo, mantiene condiciones apropiadas para G. $m$. bogotensis asociadas principalmente a la presencia de vegetación acuática ( $E$. crassipes, $H$. ranunculoides, $A$. filiculoides). A su vez, la presencia de vegetación flotante (Eichornia crassipes y Limnobium laevigatum, Lemna gibba, Azolla filiculoides) varía constantemente en los sectores "Occidente 1 y 2 " de acuerdo a las variaciones en el nivel del agua por la estacionalidad de las lluvias. Esto favorece la formación de praderas emergentes de Polygonum sp., Hydrocotile sp invadidas ocasionalmente con pasto kikuyo (K. clandestina). De tal manera que el humedal de Guaymaral le ofrece a G. m. bogotensis un mosaico de hábitats para realizar actividades de descanso, limpieza, movimiento, alimentación e interacción social. Los juncales a su vez conforman un hábitat apropiado que ofrece refugio y zonas de anidación para G. m. bogotensis (Botero-Delgadillo et al. 2006).

Estos resultados coinciden con las observaciones realizadas por De Las Casas et al. (2005) y Zuluaga-Bonilla (2007) en humedales artificiales tales como gravilleras, que se han convertido en áreas importantes que proveen hábitat disponible para Rallus semiplumbeus, Gallinula melanops, Oxyura jamaicensis, entre otras especies de aves acuáticas. Es decir, que aún cuando el área del humedal Guaymaral se ha visto reducido sustancialmente por los rellenos de terreno a su alrededor, la existencia de los estanques artificiales en los sectores "Occidente 1 y 2 " ha favorecido la presencia de G. $m$. bogotensis. Sin embargo, la supervivencia de esta población radica, igualmente, en la cercanía del humedal propiamente dicho con un cinturón de juncales definido que favorece la anidación exitosa. Los lagos artificiales, aunque son una opción para el desarrollo de la población, presentan una limitante al estar ausente la cobertura vegetal apropiada alrededor de los lugares de anidación. Por tal razón la población de G. m. bogotensis está más expuesta a la depredación, en especial por ratas (Rattus sp), tal como ha sido documentado para Gallinula chloropus en ambientes artificiales (Forman E Brain, 2004).

El seguimiento de la población de G. m. bogotensis permitió establecer cinco categorías divididas en 27 unidades comportamentales (Tabla 1). Principalmente, individuos de todas las edades se observaron forrajeando en los sectores "Bosque de la lechuza", "Occidente 1 y 2". Durante el forrajeo, los individuos se desplazaban sobre la vegetación flotante, picoteando las hojas y la raíz de $E$. crassipes, $L$. laevigatum, L. gibba y A. filiculoides. Esta actividad también se observó relacionada con las asociaciones vegetales $(H$. ranunculoides, Juncus sp. y Polygonum sp) lo que sugiere que incluyen en su alimentación bien sea otras partes vegetativas tales como semillas o pequeños artrópodos asociados a la vegetación de borde.

Durante la anidación y posterior incubación, Becerra et al. (2005) observaron que la pareja mantiene relevos de 15 a 25 minutos, vadeando en un pequeño espejo de agua, cubierto con vegetación flotante fina, principalmente, Lenteja de Agua (Lemma sp.), consumiendo, especialmente, las hojas de esta planta. En el humedal Guyamaral el desplazamiento ya sea vadeando o nadando entre las praderas emergentes es una actividad que se observó asociada tanto a E. crassipes y a otras plantas como Hydrocotile sp., Polygonum sp. y K. clandestina. Si bien los estudios desarrollados por Becerra et al. (2004) reportan a G. m. bogotensis consumiendo pasto Kikuyo, en el humedal Guaymaral la especie utiliza este tipo de vegetación en el sector "Occidente 3" solo para descansar y en el sector "Occidente 1" para acicalarse. Las vocalizaciones fueron más frecuentes en zonas cubiertas con junco (S. californicus) donde la mayoría de los sonidos se produjeron como respuesta a la presencia del observador al acercarse a la zona.

Las frecuencias obtenidas para las diferentes categorías comportamentales en este estudio indican un uso diferencial de acuerdo a la cobertura vegetal (Tabla 1). Esto corrobora lo expuesto por Estela et al. (2003), quienes observaron que las zonas de vegetación flotante son utilizadas como sitio de descanso y alimentación por las especies de la familia Rallidae, especialmente donde hay presencia de lenteja de agua ( $L$. gibba), lirio de agua ( $E$. crassipes) y buchón cucharita (L. laevigatum); mientras tanto, la franja de juncos suele ser empleada por las aves para la anidación o como refugio (ABO, 2000). Las observaciones del comportamiento de G. m. bogotensis permitieron identificar a su vez un acentuado uso de los sectores que mantienen vegetación flotante, mientras que los juncales, la ronda y las asociaciones vegetales registraron menor abundancia en todos los sectores estudiados (Figura 5). Estudios realizados en un complejo de humedales en el valle de Ubaté (Cundinamarca), registraron que la especie prefiere el hábitat de vegetación flotante con asociaciones de Bidens laevis, $L$. laevigatum, H. ranunculoides, y Myriophillum platinoides, o en áreas con espejos de agua bien diferenciados pero con presencia de Egeria densa, A. filiculoides, E. crassipes 
Tabla 1. Categorías comportamentales (CC) y Unidades Comportamentales (UC) de G. m. bogotensis en el humedal Guaymaral.

\begin{tabular}{|c|c|c|c|c|c|c|c|}
\hline \multirow{2}{*}{$\mathrm{CC}$} & \multirow{2}{*}{ UC } & \multicolumn{6}{|c|}{ FRECUENCIA/HORA/HÁBITAT } \\
\hline & & Flotante & $\begin{array}{l}\text { Praderas } \\
\text { emergentes }\end{array}$ & Juncales & Ronda & Asociaciones & $\begin{array}{l}\text { Espejo } \\
\text { de agua }\end{array}$ \\
\hline \multirow{6}{*}{ Movimiento } & $\begin{array}{c}\text { Nadar con movimiento } \\
\text { de cuello }\end{array}$ & \multirow{6}{*}{18,47} & \multirow{6}{*}{3,72} & \multirow{6}{*}{0,56} & \multirow{6}{*}{0,74} & \multirow{6}{*}{0,84} & \multirow{6}{*}{10,79} \\
\hline & $\begin{array}{c}\text { Nadar sin movimiento } \\
\text { de cuello }\end{array}$ & & & & & & \\
\hline & Caminar en la tierra & & & & & & \\
\hline & $\begin{array}{l}\text { Caminar sobre la veg- } \\
\text { etación flotante }\end{array}$ & & & & & & \\
\hline & Sumergirse & & & & & & \\
\hline & Saltar al agua & & & & & & \\
\hline \multirow{6}{*}{ Limpieza } & $\begin{array}{c}\text { Limpia con el pico la } \\
\text { espalda }\end{array}$ & \multirow{6}{*}{2,93} & \multirow{6}{*}{0,93} & \multirow{6}{*}{0,23} & \multirow{6}{*}{0,28} & \multirow{6}{*}{0,00} & \multirow{6}{*}{0,00} \\
\hline & $\begin{array}{c}\text { Limpia con el pico las } \\
\text { alas }\end{array}$ & & & & & & \\
\hline & $\begin{array}{l}\text { Limpia con el pico el } \\
\text { pecho }\end{array}$ & & & & & & \\
\hline & $\begin{array}{l}\text { Limpia las patas con el } \\
\text { pico }\end{array}$ & & & & & & \\
\hline & Baño & & & & & & \\
\hline & $\begin{array}{c}\text { Rasca la cabeza con la } \\
\text { pata }\end{array}$ & & & & & & \\
\hline \multirow{3}{*}{ Descanso } & Vigilancia & \multirow{3}{*}{1,02} & \multirow{3}{*}{0,19} & \multirow{3}{*}{0,09} & \multirow{3}{*}{0,23} & \multirow{3}{*}{0,00} & \multirow{3}{*}{0,00} \\
\hline & Descansar & & & & & & \\
\hline & Desperezarse & & & & & & \\
\hline \multirow{3}{*}{ Alimentación } & $\begin{array}{l}\text { Picoteo en agua y en } \\
\text { tierra }\end{array}$ & \multirow{3}{*}{27,26} & \multirow{3}{*}{1,49} & \multirow{3}{*}{0,28} & \multirow{3}{*}{0,14} & \multirow{3}{*}{0,84} & \multirow{3}{*}{0,00} \\
\hline & Beber agua & & & & & & \\
\hline & Captura del alimento & & & & & & \\
\hline \multirow{4}{*}{ Vocalización } & Canto territorial & \multirow{4}{*}{0,93} & & & & & \\
\hline & Canto & & 074 & 126 & 000 & 005 & 009 \\
\hline & Vocaliza y corre & & & & & & \\
\hline & Canta y sacude & & & & & & \\
\hline
\end{tabular}

(IAvH \& Fundación Humedales, 2004). El uso diferencial de la vegetación acuática presente en el hábitat igualmente se refleja en la elaboración de los nidos, para los cuales G. $m$. bogotensis utiliza principalmente la Gualola (Polygonum segetum) como base y techo y la Hierba de sapo (Polygonum hidropiperoides), la Sombrillita de agua (H. ranunculoides), el Junco (S. californicus) y el Kikuyo para entretejer el nido a manera de canasta (Becerra et al. 2005).

La presencia permanente de G. $m$. bogotensis en el humedal 


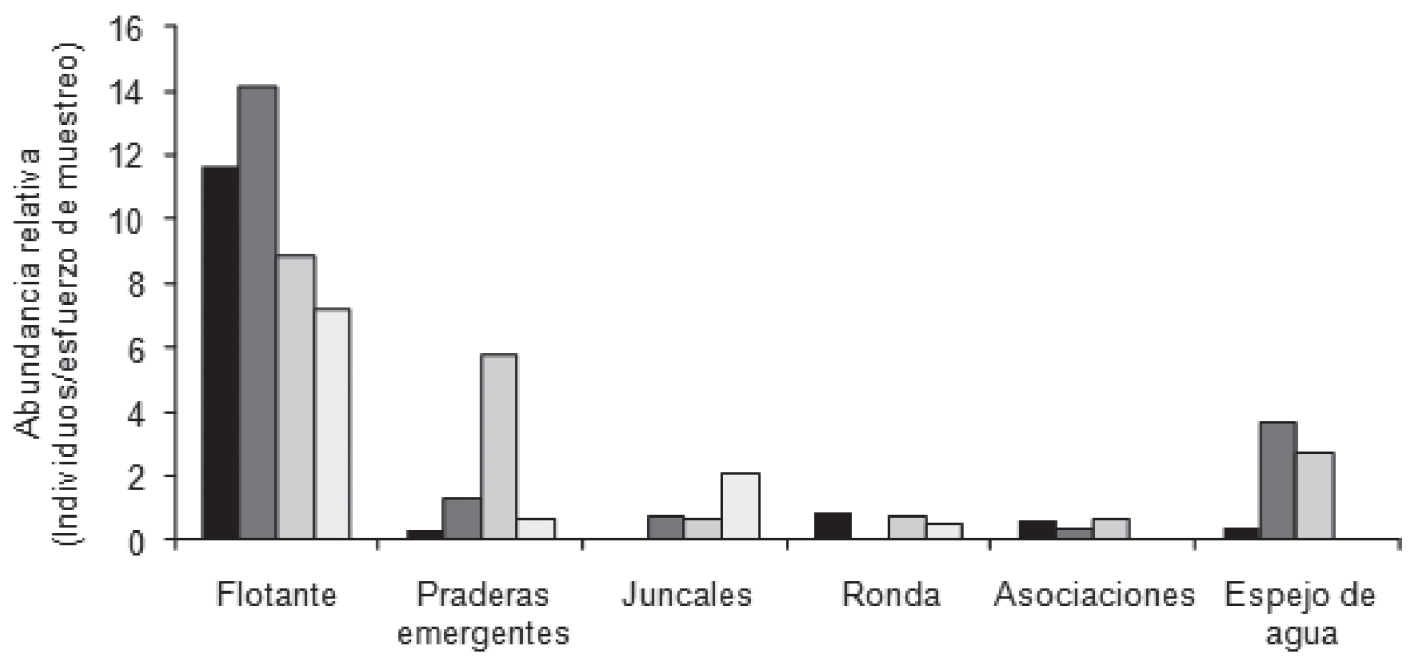

Bosque de la lechuza $\quad \square$ Occidente $1 \quad \square$ Occidente $2 \quad \square$ Occidente 3

Figura 5. Proporción de uso de las coberturas vegetales presentes en los diferentes sectores del humedal Guaymaral por parte de G. $m$. bogotensis entre 2008 y 2010. Flotante $=$ E. crassipes; $L$. laeviegatum; Asociaciones $=$ L. laevigatum $-H$. ranunculoides; L. laevigatum - Polygonum sp.; $L$. laevigatum - Ciperaceae; L. laevigatum - Juncus sp.; Pradera emergente = Polygonum sp.; H. ranunculoides Ronda = Kikuyuochloa clandestina; H. bonplandii; Juncal = S. californicus; $T$. angustifolia; J. effusus.

Guaymaral corrobora que la especie puede adaptarse a los efectos de la actividad humana, siempre y cuando subsistan zonas con juncales que favorezcan la anidación (CIC \& EAAB 2000; Rueda-C et al. 2005). Sin embargo, la perturbación sostenida también produce cambios conductuales afectando el éxito reproductivo, tiempo de alimentación o la tolerancia a la presencia humana (Kusch et al. 2008). Por tal razón, es recomendable evitar la pérdida continua de la vegetación original en el humedal Guaymaral y mejorar las condiciones de hábitat a través de la restauración ecológica en los cuerpos de agua artificiales.

Conflicto de intereses: Hacemos constar que el manuscrito fue revisado y preparado por los autores quienes declaran que no existe ningún conflicto de intereses que ponga en riesgo los resultados presentados. Financiación: Este estudio fue financiado por Zoological Society for the Protection of Species and Populations (ZGAP), el Programa Jóvenes Investigadores e Innovadores COLCIENCIAS y la U.D.C.A.

\section{BIBLIOGRAFÍA}

1. ASOCIACION BOGOTANA DE ORNITOLOGIA (ABO). 2000. Aves de la Sabana de Bogotá - Guía de campo. ABO-CAR, Bogotá, Colombia. 276p.
2. ARZUZA, D.E.; MORENO, M.I.; SALAMAN, P. 2008. Conservación de las aves acuáticas en Colombia. Conservación Colombiana. 6:1-72.

3. BECERRA, L.F.; PATIÑO, M.; BENITEZ, H. 2004. Humedales bogotanos: ecosistemas, biodiversidad y conservación. Rev. Cient. U. Distrital Francisco José de Caldas. 6:37-41.

4. BECERRA, L.F.; BENITES, H.D; CELI, J.E.; PATIÑO, L. 2004a. Reproducción, alimentación y comportamiento de la polla sabanera Gallinula melanops bogotensis en tres humedales de la Sabana de Bogotá. Disponible desde Internet en: http://www. ornitologiacolombiana.org/MemoriasICOC/posters/ becerraetal.htm (con acceso el 24/01/11).

5. BECERRA, L.F.; BENITES, H.D; CELY, J.E; PATIÑO, L. 2005. Notas sobre la anidación no exitosa de la Tingua Moteada (Gallinula melanops) en un canal artificial del humedal Jaboque, Bogotá. Boletín SAO. 15(1):29-38.

6. BECERRA G., L.F.; CELY-FAJARDO, J. 2007. Análisis genético poblacional y de endogamia para Gallinula melanops, (Aves: Rallidae) en poblaciones de los departamentos de Cundinamarca y Boyacá. Mem. 
$2^{\circ}$ Congreso Colombiano de Ornitología, U.N. Colombia, Bogotá, 75p.

7. BIBBY, C.; JONES, M.; MARSDEN, S. 1998. Expedition Field Techniques Bird Surveys. Expedition Advisory Centre Royal Geographical Society, London, 143p.

8. BORRERO, J.I. 1956. Apuntes sobre aves colombianas. Lozanía (Acta Zoológica Colombiana) 3:1-12.

9. BOTERO-DELGADILLO, E.; AGUILERA-GONZÁLEZ, A.M.; VERHELST, J.C. 2006. Abundancia, riqueza y diversidad de la avifauna presente en la Estación Experimental de la Facultad de Ciencias de la Universidad Militar Nueva Granada (Cajicá, Colombia). Rev. Fac. Cienc. Bás. 2(1):165-175.

10. CADENA, C.D. 2002. Gallinula melanops. In: Rengifo L.M.; Franco-Maya, A.M.; Amaya-Espinel, J.D.; Kattan, G.; López-Lanus, B. (eds.). Libro rojo de Aves de Colombia. Serie Libros rojos de especies amenazadas de Colombia. Instituto de Investigación de Recursos Biológicos Alexander Von Humboldt y Ministerio del Medio Ambiente, Bogotá, Colombia. p.173-177.

11. CIFUENTES, Y.; CASTILLO-CORTÉS, L.F. 2009. Colombia: informe anual. Censo Neotropical de Aves Acuáticas 2008. En: Unterkofler D.A.; Blanco, D.E. (eds.): El Censo Neotropical de Aves Acuáticas 2008; Una herramienta para la conservación. Wetlands International, Buenos Aires, Argentina. Disponible desde internet en http:/lac.wetlands.org/ (con acceso 26/01/11).

12. CONSERVACIÓN INTERNACIONAL COLOMBIA (CIC); Empresa de Acueducto y Alcantarillado de Bogotá (EAAB). 2000. Protocolo General de Revegetalización en los Humedales Bogotanos. Bogotá, Colombia. 80p.

13. CONSERVACIÓN INTERNACIONAL COLOMBIA (CIC). 2003. Fichas resumen sobre el estado actual de los humedales de Bogotá. En: Los humedales de Bogotá y la sabana. Tomo 2. Acueducto de Bogotá Colombia. p.250-272.

14. DEPARTAMENTO ADMINISTRATIVO DEL MEDIO AMBIENTE, DAMA. 2006. Política de humedales del Distrito Capital. Alcaldía Mayor de Bogotá, Bogotá, D. C., Colombia. 73p.

15. DE LAS CASAS, J.C.; DEVENISH, C.; RUIZ, J.M.; GALLEGO, N.I.; VALENCIA, I.D. 2005. Conservación de las gravilleras del valle del río Siecha. Asociación Bogotana de Ornitología - ABO, 74p.

16. ESTELA, F.A.; HERNÁNDEZ-CORREDOR, C.E.; FALKFERNÁNDEZ, P.E.; CHÁVES, P. 2003. Caracterización ambiental preliminar de los humedales de la cuenca del Rio Cauca, Departamento del Cauca. Asociación Calidris. Cali, Colombia. 35p

17. FORMAN, D.W.; BRAIN, P.F. 2004. Reproductive strategies used by moorhens (Gallinula chloropus) colonizing an artificial wetland habitat in south Wales. J. Nat. Hist. 38:389-401.

18. FRANCO, A.M.; BRAVO, G. 2005. Áreas Importantes para la Conservación de Aves en Colombia. In: BirdLife International y Conservación Internacional. Áreas Importantes para la Conservación de las Aves en los Andes Tropicales: sitios prioritarios para la conservación de la biodiversidad. BirdLife Ecuador, BirdLife International (Serie de Conservación de BirdLife). 14:117-281

19. GUARNIZO, A.; CALVACHI, B. 2004. Los humedales de Bogotá y la Sabana. Tomos I y II Publicación del acueducto de Bogotá y Conservación Internacional Colombia. 262p.

20. HERNÁNDEZ, S.; ANDRADE, G. 2006. Biodiversidad, introducción a su conocimiento, conservación y manejo sostenible en las lagunas de Fúquene, Cucunubá y Palacio. Valle de Ubaté. Colombia. Fundación Humedales Serie Divulgación Técnica. 3:1-32.

21. HILTY, S.L.; BROWN, W.L. 1986. A guide to the birds of Colombia. Princeton University Press. Princeton, New Jersey. USA. 410p.

22. HUTT, S.J.; HUTT, C. 1970. Direct observation and measurement of behavior. Charles C. Thomas Springfield, IL. 224p.

23. INSTITUTO DE INVESTIGACIÓN DE RECURSOS BIOLÓGICOS ALEXANDER VON HUMBOLDT (IAvH); FUNDACIÓN HUMEDALES. 2004. Caracterización biofísica, ecológica y sociocultural del complejo de humedales del Valle de Ubaté: Fúquene, Cucunubá y Palacio. 214p.

24. KUSCH, A.; CÁRCAMO, J.; GÓMEZ, H. 2008. Aves acuáticas en el humedal urbano de Tres Puentes, Punta Arenas (53 5 ), Chile austral. Anales Instituto Patagonia (Chile). 36(2):45-51. 
25. LEHNER, P. 1996. Handbook of ethological methods, Cambridge University Press. 671p.

26. LOZANO, I.E. 1992. La tingua de los pantanos. Ecologica. 3(13):20-21.

27. MATALLANA, E.; CÁRDENAS, C.; BORRERO, I.; VANEGAS, C. 2004. Atlas Ambiental Localidad 11, Suba, Unidad de Gestión Ambiental, Alcaldía Local de Suba. 112p.

28. MOSTACEDO, B.; FREDERICKSEN, T.S. 2000. Manual de métodos básicos de muestreo y análisis en Ecología vegetal. Proyecto de manejo forestal sostenible, BOLFOR, Santa Cruz, Bolivia. 92p.

29. OSBAHR, K.; GÓMEZ, N. 2006. Uso de hábitat de la avifauna en el humedal Guaymaral (Cundinamarca). Rev. U.D.C.A Act. \& Div. Cient. 9(2):157-168.

30. OSBAHR, K.; HERNÁNDEZ-SCHMIDT, M. 2006. Caracterización florística de un fragmento del humedal Torca - Guaymaral (Bogotá-Cundinamarca). Rev. U.D.C.A Act. E Div. Cien. 9(1):52-63

31. OSBAHR, K.; WÜST, R. 2009. Das seltene BogotáMaskenpfuhlhuhn (Gallinula melanops bogotensis). ZGAP Mitteilungen. 25(2):6-8.

32. RALPH, C.J.; GEUPEL, G.R.; PYLE, P.; MARTIN, T.E.; DESANTE, D.F.; BORJA, M. 1996. Manual de métodos de campo para el monitoreo de aves terrestres. Gen. Tech. Rep. PSW-GTR-159. Albany,CA: Pacific Southwest Research Station, Forest Service, U.S. Department of Agriculture, 46p.

33. RENJIFO, L.M. 1998. Especies de aves amenazadas y casi amenazadas de extinción en Colombia. In: Informe nacional sobre el estado de la biodiversidad en Colombia. Tomo 1: Diversidad biológica. Chávez, M.E.; Arango, N. (Eds.). Instituto de Investigación de Recursos Biológicos Alexander von Humboldt, PNUMA, Ministerio del Medio Ambiente, Bogotá, Colombia. p.416-426.

34. RESTALL, R.; RODNER, C.; LENTINO, M. 2007. Birds of Northern South America: Species accounts. Yale University Press. 880p.
35. RUEDA-C, L.E.; ZERDA-O, E.; DEL VALLE-U, C.M.; HERNÁNDEZ-B, V. 2005. Uso de hábitat de la avifauna del humedal Jaboque, localidad de Engativá, Bogotá, D.C., Colombia. Convenio investigación aplicada en restauración en el humedal de Jaboque, Instituto de Ciencias Naturales Universidad Nacional, Bogotá. 32p.

36. SCOTT, D.A.; CARBONELL, M. 1986. Inventario de Humedales de la Región Neotropical. International Waterfowl Research Bureau (IWRB) Slimbridge, UICN, Cambridge, UK. 95p.

37. STILES, F.G. 1998. Las aves endémicas de Colombia. In: Informe nacional sobre el estado de la biodiversidad en Colombia. Tomo 1: Diversidad biológica. Chávez, M.E.; Arango, N. (Eds.). Instituto de Investigación de Recursos Biológicos Alexander von Humboldt, PNUMA, Ministerio del Medio Ambiente, Bogotá, Colombia. p.378-385, 428-432.

38. VARTY, N.J.; ADAMS, J.; ESPIN, P.; HAMBLER, C. 1986. An ornithological survey of Lake Tota, Colombia. International Council for Bird Preservation (Study Report 12), Cambridge, UK. 87p.

39. VAN DER HAMMEN, T.; STILES, F.G.; ROSSELLI, L.; CHISACÁ HURTADO, M.L.; CAMARGO PONCE DE LEÓN, G.; GUILLOT MONROY, G.; USECHE SALVADOR, Y.; RIVERA OSPINA, D. 2008. Protocolo de recuperación y rehabilitación ecológica de humedales en centros urbanos. Secretaría Distrital de Ambiente, Bogotá, 300p.

40. ZAR, J.H. 1999. Biostatistical analysis. 4th Edition. Prentice Hall Inc. New Jersey. U.S.A. 663p.

41. ZULUAGA-BONILLA, J.E. 2007. Registros de reproducción de Gallinula melanops bogotensis en un lago artificial. Finca "El Capitolio". Tunja, Boyacá. Mem. $2^{\circ}$ Congreso Colombiano de Ornitología, U.N. de Colombia, Bogotá, 75p.

Recibido: Marzo 8 de 2011

Aceptado: Mayo 4 de 2011 